\title{
Long non-coding RNA FOXF1 adjacent non-coding developmental regulatory RNA inhibits growth and chemotherapy resistance in non-small cell lung cancer
}

Ran Xu, Yun Han

Department of Thoracic Surgery, Shengiing Hospital, China Medical University, Shenyang, Liaoning, China

Submitted: 26 February 2017

Accepted: 4 July 2017

Arch Med Sci 2019; 15 (6): 1539-1546

DOI: https://doi.org/10.5114/aoms.2019.86707

Copyright $@ 2019$ Termedia \& Banach

\section{Abstract}

Introduction: Lung cancer is one of the most common malignant neoplasms around the globe. Its most common type is non-small cell lung cancer (NSCLC). The FOXF1 adjacent non-coding developmental regulatory RNA (FENDRR) gene is an IncRNA which has been reported to show low expression and a tumor suppressor role in NSCLC.

Material and methods: The expression of FENDRR in NSCLC patients' tissues and cell line was detected by quantitative real-time PCR. MTT assay was used to detect cell proliferation and chemotherapy resistance. Cell apoptosis was measured by flow cytometry.

Results: The expression of FENDRR was low in NSCLC tissues and cells in contrast to control tissues and cells, and low FENDRR expression correlated with high TNM stages and poor differentiation of NSCLC, and could be a promising prognostic factor for NSCLC. FENDRR enhancement could inhibit the proliferation ability and advance cell apoptosis of A549 cells. The expression of FENDRR in NSCLC tissues and cells insensitive to cisplatin was much lower than that in NSCLC tissues and cells sensitive to cisplatin. The chemotherapy resistance to cisplatin of A549/DDP cells was depressed by FENDRR enhancement, and IC50 for cisplatin presented a conspicuous depression. FENDRR up-regulation inhibited cell viability of A549/DDP cells under treatment with $5 \mu \mathrm{g} / \mathrm{ml}$ DDP. TCGA Pan-Cancer (PANCAN) showed that the expression of FENDRR was negatively correlated with the expression of $A B C C 10$ in lung cancer, and our western blot found that FENDRR up-regulation inhibited the expression of ABCC10 in A549/DDP cells.

Conclusions: LncRNA FENDRR has low expression in NSCLC and functions as a potential tumor-suppressing gene to inhibit growth and chemotherapy resistance of NSCLC cells.

Key words: long non-coding RNA, FENDRR, non-small cell lung cancer, chemotherapy resistance, cisplatin.

\section{Introduction}

Lung cancer is one of the most common malignant neoplasms around the globe, its most common type being non-small cell lung cancer (NSCLC) [1]. The main therapy strategies for NSCLC include surgical operation, systemic radiotherapy and chemotherapy, which could effectually pre-

\author{
Corresponding author: \\ Prof. Yun Han \\ No. 36 Sanhao St \\ Heping Area \\ Shenyang 100004, China \\ Phone/fax: +86-24-96615 \\ E-mail: \\ submissioncmu@163.com
}


vent metastasis and recrudescence of NSCLC [2]. Despite prominent advances in modern surgical technique and systemic chemotherapy, the prognosis of NSCLC patients is still poor; its 5-year survival rate is less than 15\% [3]. Consequently, it is urgent to study the molecular mechanisms involved in the tumorigenesis and development of NSCLC, which will contribute to the progress of NSCLC's clinic therapy.

Long noncoding RNA (IncRNA) is a category of endogenous non-coding RNAs longer than 200 nucleotides, which is a hotspot in current research. By January 2016, 294 IncRNAs had been functionally annotated in the LncRNAdb database; most of them had been found and researched in human. LncRNAs have various functions and complicated regulative mechanisms, and take part in almost all physiological or pathological cytobiological characteristics, including apoptosis, cell proliferation and chemotherapy resistance [4-7].

The FOXF1 adjacent non-coding developmental regulatory RNA (FENDRR) gene is an IncRNA located at 16q24.1, which produces a spliced long non-coding RNA transcribed bidirectionally with FOXF1 on the opposite strand. To date, limited data about the aberrant expression and functions in cancers have been reported. Xu et al. reported that the FENDRR gene showed low expression in gastric cancer and participate in the modulation of metastasis and progression, and its low expression predicted poor prognosis of gastric cancer patients [8]. In Li's microarray assays, the expression of FENDRR showed significant down-regulation in lung cancer [9]. Those discoveries suggested that FENDRR might also play a tumor-suppressing role in NSCLC, but its role and clinical significance in NSCLC is still unclear.

In this study, the expression of FENDRR was confirmed to be low in NSCLC tissues in contrast to control tissues, and low FENDRR expression correlated with high TNM stages and poor differentiation of NSCLC. Moreover, FENDRR played its tumor-suppressing role to inhibit cell proliferation ability and chemotherapy resistance, and advance cell apoptosis of NSCLC cells. The consequences will contribute to mechanism research and clinical treatments for NSCLC.

\section{Material and methods}

\section{Clinical specimens}

All 74 NSCLC and normal lung tissues (NLT) specimens were obtained in the Department of Thoracic Surgery, Affiliated Shengjing Hospital of China Medical University (Shenyang, China) from April 2009 to November 2010. Another 50 NSCLC specimens were obtained from October 2012 to
June 2013 through bronchoscopy, and treated with cisplatin. According to RECIST Response Evaluation Criteria, these NSCLC patients were divided into two groups according to the sensitivity to cisplatin: the sensitive group $(n=21)$ was completely or partially sensitive to cisplatin, while the insensitive group $(n=29)$ was insensitive to the cisplatin. The clinical pathological data were confirmed by a pathologist according to WHO standards, and follow-up continued 5 years until December 2015. This study was approved by the Ethics Committees of China Medical University.

\section{Cell culture}

Human pulmonary alveolar epithelial cells (PAEC) were obtained from ScienCell Research Laboratories (Santiago, Ca, USA). Human NSCLC A549 cells were obtained from the Chinese Academy of Medical Sciences (Beijing, China). The A549/DDP cell line was obtained from Runcheng Company (Shanghai, China), which showed resistance to cisplatin. The cells were cultured in RPMI-1640 medium with $10 \%$ fetal bovine serum (Gibco, Carlsbad, CA, USA) at $37^{\circ} \mathrm{C}$. In order to maintain the resistance phenotype, A549/DDP cells was cultured with $5.0 \mu \mathrm{g} / \mathrm{ml}$ cisplatin (Sigma, St. Louis, MO, USA), and cultured in cisplatin-free medium for 10 days prior to the experiment.

\section{Quantitative real-time PCR (qRT-PCR)}

Then FENDRR expression level was detected with SYBR (Applied Biosystems, Foster City, CA, USA); its forward primer was 5'-TAAAATTGCAGATCCTCCG-3' and the reverse primer was 5'-AACGTTCGCATTGGTTTAGC-3'. GAPDH was used as a reference gene; its forward primer was 5'-GTCAACGGATTTGGTCTGTATT-3' and the reverse primer was 5'-AGTCTTCTGGGTGGCAGTGAT-3'. The $2^{-\Delta \Delta C t}$ method was used to quantify the relative expression of FENDRR [10].

\section{Transfection}

Various vectors were transfected with Lipofectamine 3000 Reagent (Invitrogen, Foster City, CA, USA) according to the operation manual. After $4 \mathrm{~h}$, normal medium was applied to cultured cells for $48 \mathrm{~h}$.

\section{Vector construction}

The FENDRR expression vector pc-FENDRR was constructed by Sangon Company (Shanghai, China). The pcDNA3.1 vector (Invitrogen, Carlsbad, CA, USA) was selected to be the negative control (pc-NC). 


\section{Cell proliferation assay}

The cells $\left(2 \times 10^{4} /\right.$ well $)$ were seeded in a 96-well plate. $20 \mu \mathrm{l}$ of MTT solution $(0.5 \mathrm{mg} / \mathrm{ml}$, Sigma, St. Louis, MO, USA) per well was added to the 96-well plate, and it was incubated at $37^{\circ} \mathrm{C} .4 \mathrm{~h}$ later, MTT solution was discarded, and to each well $0.2 \mathrm{ml}$ was added, then it was incubated for $30 \mathrm{~min}$. For OD values and the calculation of cell viability refer to the previous literature [11].

\section{Apoptosis detection}

Flow cytometry was used to examine the apoptosis rate with the Annexin V-FITC apoptosis test kit (Beyotime, Nantong, Jiangsu, China) according to the operation manual, and the data were analyzed through CellQuest 3.0 software (BD, Franklin Lakes, NJ, USA).

\section{Western blot}

Protein was extracted by lysing with RIPA buffer (Beyotime, Shanghai, China) and the concentration was determined using the BCA assay (Beyotime, Shanghai, China) according to the manufacturer's instructions. A total of $30 \mu \mathrm{g}$ of protein were subjected to $10 \%$ SDS-PAGE and transferred to a $0.22 \mu \mathrm{m}$ PVDF membrane. After being incubated with specific ABCC10 antibodies (ab91451, Abcam, USA) on a shaker overnight at $4^{\circ} \mathrm{C}$, signals were visualized using the infrared labeled antibody and scanned with the Dual Color Infra-red Laser Imaging System (Gene, HK, China) by being normalized to the inference gene of GAPDH.

\section{Chemotherapy resistance assay}

Cells were treated with cisplatin at various concentrations $(1.0 \mu \mathrm{g} / \mathrm{ml}, 5.0 \mu \mathrm{g} / \mathrm{ml}, 10 \mu \mathrm{g} / \mathrm{ml}, 15 \mu \mathrm{g} / \mathrm{ml}$, $20 \mu \mathrm{g} / \mathrm{ml}, 25 \mu \mathrm{g} / \mathrm{ml}$ ) [11]. The cell viability was detected by MTT assay $48 \mathrm{~h}$ later and the dose-re- sponse curve charted. The half maximal inhibitory concentration $\left(\mathrm{IC}_{50}\right)$ was calculated by the Probit regression model.

\section{Statistical analysis}

All experiments were done 5 times. SPSS 21.0 software (IBM, Somers, NY, USA) was selected to perform the statistical analysis. For the comparison of differences piared Student's $t$-test was used. The association between FENDRR expression and pathological grades was assessed using one-way ANOVA and binary logistic regression. The overall survival rate was calculated by the Kaplan-Meier method. $P<0.05$ means a significant difference.

\section{Results}

FENDRR showed low expression in NSCLC specimens and cells

Figure 1 A shows that the FENDRR expression in NSCLC was much lower than that in NLT $(p<0.05)$. Moreover, compared with control PAEC cells, the FENDRR expression was down-regulated in A549 cells, and down-regulated much more in A549/DDP cells ( $p<0.05$, Figure $1 \mathrm{~B}$ ). Those results suggested that FENDRR might play a role in genesis and chemotherapy resistance of NSCLC.

The correlations between FENDRR expression and clinical pathological characteristics of NSCLC were investigated using univariate and multivariate analysis. Lower FENDRR expression in NSCLC tissues occurred much more often in patients with more malignant behaviors, including high TNM stage and poor differentiation $(p<0.05$, Table I), but excluding age, sex and lymphatic metastasis. Those results provided initial evidence that FENDRR might be involved in genesis and progression of NSCLC, but not in metastasis.
A

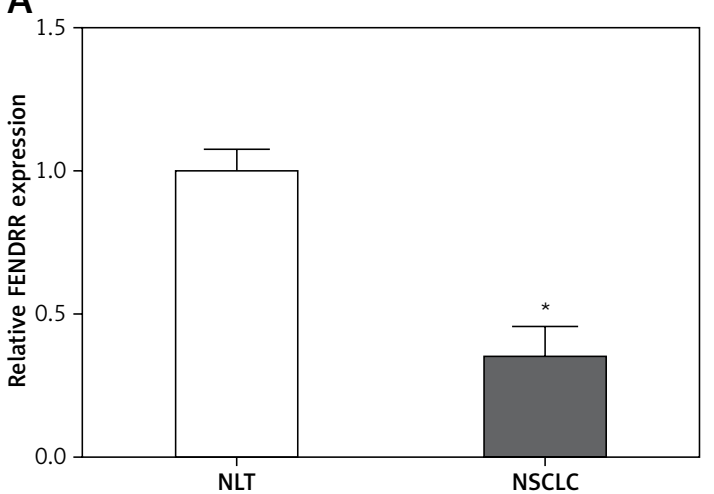

B

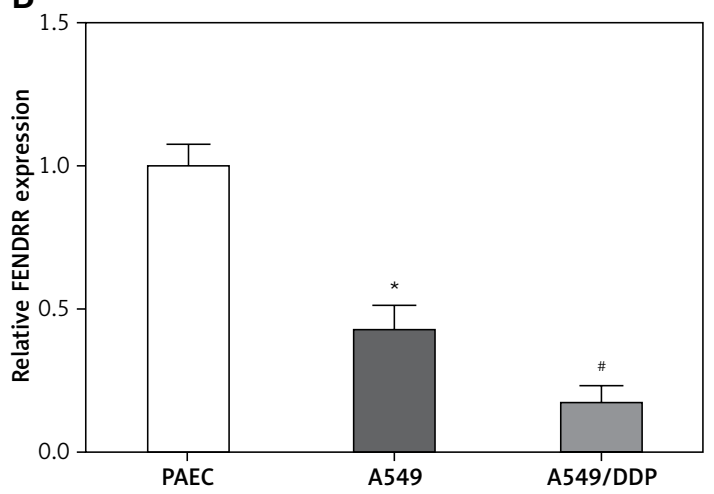

Figure 1. FENDRR showed low expression in NSCLC specimens and cells. A - Relative expression of FENDRR showed low expression in NSCLC tissues $(n=74)$. ${ }^{*} p<0.05$ vs. NLT. B - FENDRR expression in A549 cells was higher than that in control PAEC cells, and its expression in A549/DDP cells was even much higher. ${ }^{*} p<0.05$ vs. PAEC, $p<0.05$ vs. A549 
Table I. Univariate analysis of the association between FENDRR expression and clinical pathological factors in 74 NSCLC patients

\begin{tabular}{|c|c|c|c|c|}
\hline Factor & & $N$ & Mean \pm SD & $P$-value \\
\hline \multirow[t]{2}{*}{ Age } & $\leq 58$ & 37 & $0.34 \pm 0.05$ & 0.2013 \\
\hline & $>58$ & 37 & $0.32 \pm 0.08$ & \\
\hline \multirow[t]{2}{*}{ Sex } & Male & 45 & $0.32 \pm 0.06$ & 0.0531 \\
\hline & Female & 29 & $0.35 \pm 0.07$ & \\
\hline \multirow[t]{2}{*}{ Differentiation } & Well/moderately & 48 & $0.38 \pm 0.08$ & $<0.01^{*}$ \\
\hline & Poorly & 26 & $0.24 \pm 0.04$ & \\
\hline \multirow[t]{2}{*}{ Lymphatic metastasis } & Negative & 30 & $0.35 \pm 0.09$ & 0.0893 \\
\hline & Positive & 44 & $0.32 \pm 0.06$ & \\
\hline \multirow[t]{2}{*}{ TNM stage } & $I+I \mid$ & 47 & $0.37 \pm 0.07$ & $<0.01^{*}$ \\
\hline & III + IV & 27 & $0.26 \pm 0.03$ & \\
\hline
\end{tabular}

*The difference is significant.

FENDRR was a prognostic factor for NSCLC patients

NSCLC patients with higher FENDRR expression had a remarkably longer survival time than patients with lower FENDRR expression. The overall survival rate of 5 year is 10.8 percent in the higher FENDRR expression group, but $29.7 \%$ in the lower group. Meanwhile, patients with lower expression of FENDRR had a significantly worse prognosis according to a univariate Cox proportional hazards regression analysis of overall survival $(H R=0.5818$, $95 \% \mathrm{Cl}: 0.3450-0.9811, p=0.042)$. Those outcomes verified that low-expressed FENDRR participated in the progression of NSCLC.

\section{Up-regulation of FENDRR inhibited the proliferation ability and advanced cell apoptosis in A549 cells}

To investigate the influence of FENDRR expression changes on the cell characteristics of NSCLC cells, the expression vector for FENDRR was transfected into A549 cells to up-regulate the expression of FENDRR (Figure $2 \mathrm{~A}$ ). After transfection, compared with the control groups, the cell viability of A549 cells with FENDRR over-expression decreased significantly (Figure 2 B). Moreover, the results of apoptosis detection showed that the apoptosis rate of A549 cells with FENDRR over-expression was much higher than that in the control groups (Figure 2 C).

\section{FENDRR was correlated with cisplatin} resistance of NSCLC patients

Cisplatin is one of the first-line drugs applied in chemotherapy for NSCLC patients. In this study, 50 NSCLC patients treated with chemotherapy were divided into two groups according to the sensitivity to cisplatin, and the expression of FENDRR in the insensitive group was much lower than that in the sensitive group (Figure $3 \mathrm{~A}$ ). This result provided initial evidence that FENDRR down-regulation might be involved in chemotherapy resistance of NSCLC.

The $I C_{50}$ values of cisplatin in A549 and A549/ DDPcellswere $11.38 \pm 1.17 \mu \mathrm{g} / \mathrm{mland} 26.41 \pm 1.53 \mu \mathrm{g} /$ $\mathrm{ml}$, and A549/DDP cells showed stronger resistance to cisplatin ( $p<0.05$, Figure $3 \mathrm{~B}$ ). The expression of FENDRR was up-regulated also by transfection with pc-FENDRR in A549/DDP cells ( $p<0.05$, Figure 3 C). Those results revealed that the expression of FENDRR was positively correlated with the response of patients to cisplatin-associated chemotherapy.

\section{Up-regulation of FENDRR inhibited chemotherapy resistance to cisplatin in A549/DDP cells}

Figure 3 B shows that FENDRR enhancement could depress IC50 of cisplatin from 26.41 $\pm 1.53 \mu \mathrm{g} / \mathrm{ml}$ to $15.62 \pm 1.25 \mu \mathrm{g} / \mathrm{ml}$ in A549/DDP cells $(p<0.05)$, which demonstrates that overexpression of FENDRR inhibits chemotherapy resistance to cisplatin in NSCLC cells. Moreover, the A549/DDP cells were treated with DDP $(5 \mu \mathrm{g} / \mathrm{ml})$ and the MTT assay indicated that FENDRR up-regulation inhibited cell viability of A549/DDP cells (Figure $3 \mathrm{D}, p<0.05$ ).

Up-regulation of FENDRR inhibited expression of ATP binding cassette subfamily $C$ member 10 (ABCC10) in A549/DDP cells

The co-expression patterns (log2-scale) between FENDRR and ABCC10 in TCGA Pan-Cancer (PANCAN) 
A

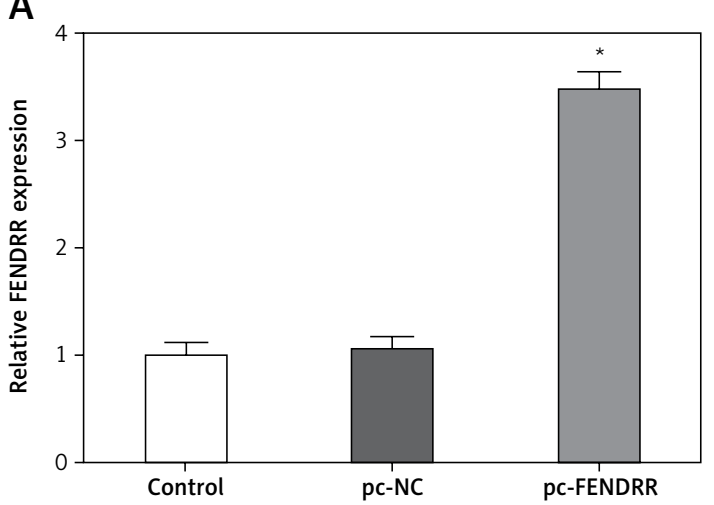

B

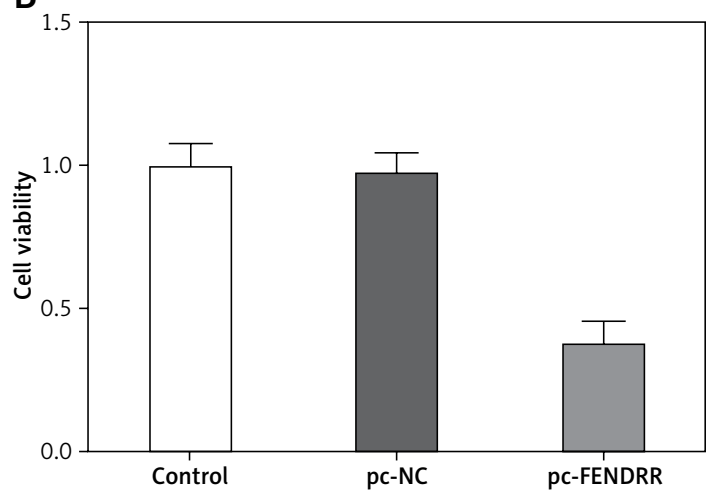

C
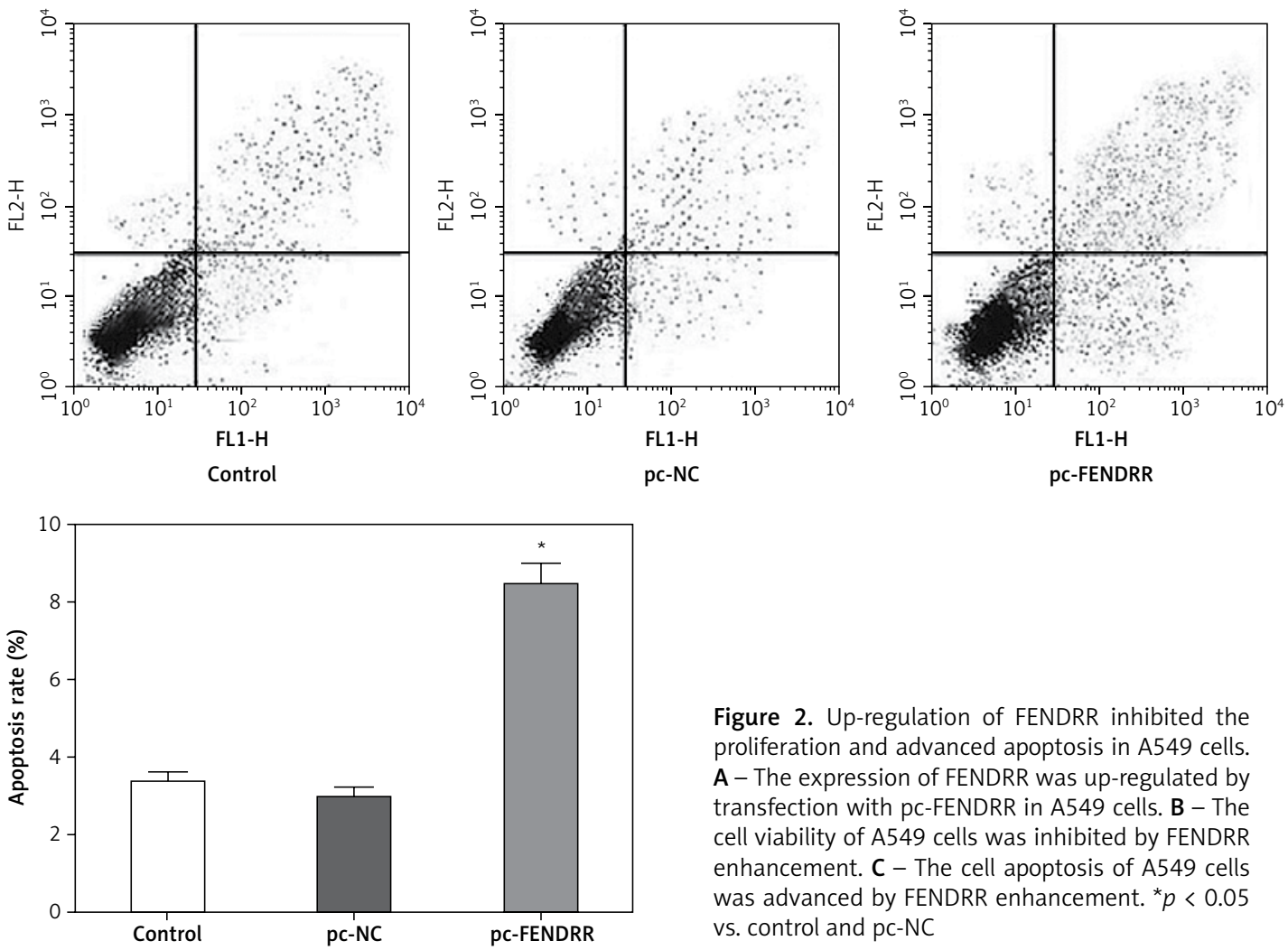

Figure 2. Up-regulation of FENDRR inhibited the proliferation and advanced apoptosis in A549 cells. A - The expression of FENDRR was up-regulated by transfection with pc-FENDRR in A549 cells. B - The cell viability of $A 549$ cells was inhibited by FENDRR enhancement. C - The cell apoptosis of A549 cells was advanced by FENDRR enhancement. ${ }^{*} p<0.05$ vs. control and pc-NC

showed that the expression of FENDRR was negatively correlated with the expression of ABCC10 (Figures $4 \mathrm{~A}, \mathrm{~B})$, with a Pearson coefficient $r=-0.2167$ and $p$-value (two tail, $t$-test) $=1.59 \times 10^{-7}$ in lung adenocarcinoma (LUAD), and a Pearson coefficient $r=-0.2014$ and $p$-value (two tail, $t$-test) $=2 \times 10^{-6}$ in lung squamous cell carcinoma (LUSC).

Also, the western blot showed that FENDRR up-regulation inhibited the expression of ABCC10, a multidrug resistance associated protein, in A549/DDP cells (Figure 4 C, $p<0.05$ ).

\section{Discussion}

LncRNAs are hotspots in current research, in which anomalous expression of certain IncRNAs is found in a variety of malignant cancers, and those IncRNAs can act as tumor-suppressing genes or oncogenes to play a part in genesis and progression of tumors $[12,13]$. It is well known that InCRNAs possess various functions and are involved in almost all cancer cell characteristics, including apoptosis, invasion, autophagy and chemotherapy resistance [14-19].

Recently, FENDRR has been reported to show low expression in 5 cell lines and tumor tissues of gastric cancer. Its low expression correlated with higher tumor stage, deeper tumor invasion and lymphatic metastasis, which suggested that FENDRR participates in the modulation of metastasis and progression of gastric cancer [8]. In our study, we confirmed that FENDRR showed low ex- 
A

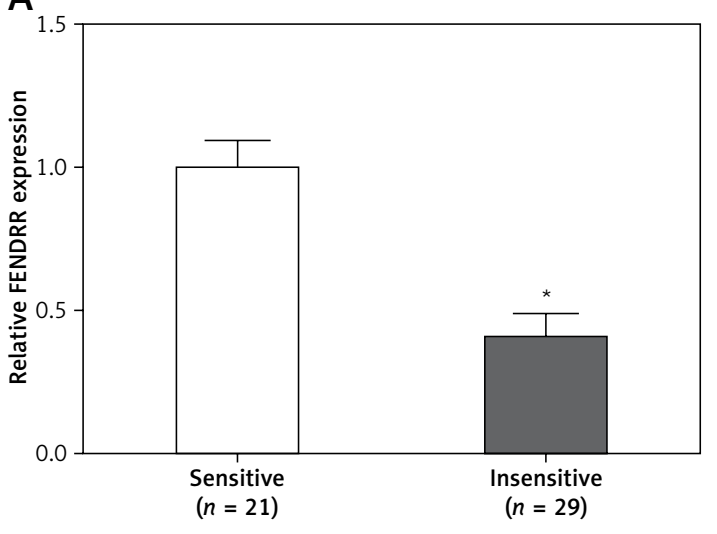

C

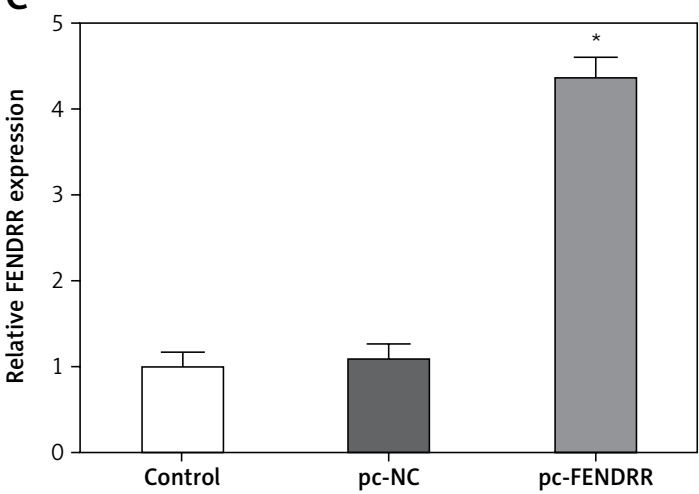

B

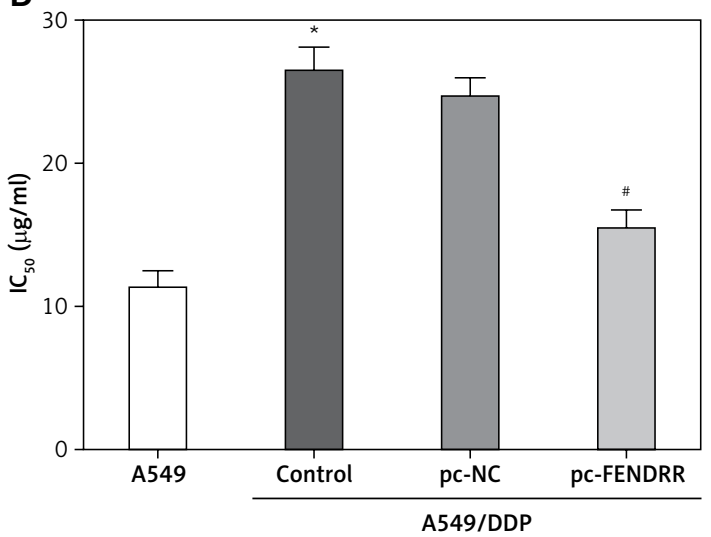

D

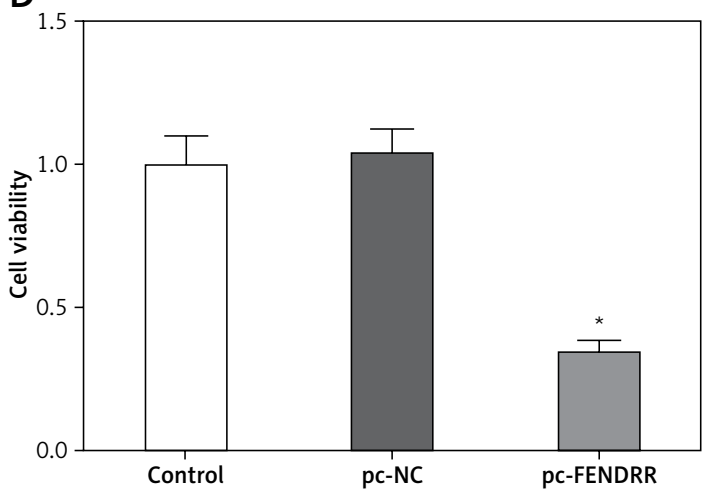

Figure 3. FENDRR was correlated with cisplatin resistance of NSCLC patients and inhibited cisplatin resistance in A549/DDP cells. A - The expression of FENDRR in NSCLC patients insensitive to cisplatin $(n=21)$ was much lower than that in NSCLC patients sensitive to cisplatin $(n=29)$. ${ }^{*} p<0.05$ vs. sensitive. B - The cisplatin concentration of $50 \%$ inhibition of cell growth (IC $\mathrm{C}_{50}$ ) of A549/DDP cells was much higher than that in A549 cells, and FENDRR enhancement depressed IC ${ }_{50}$ of doxorubicin in A549/DDP cells. ${ }^{*} p<0.05$ vs. A549, ${ }^{*} p<0.05$ vs. control. C - The expression of FENDRR was up-regulated by transfection with pc-FENDRR in A549/DDP cells. ${ }^{*}<<0.05$ vs. control and pc-NC. D - FENDRR up-regulation inhibited cell viability of A549/DDP cells under treatment with DDP (5 $\mu \mathrm{g} / \mathrm{ml})$. ${ }^{*} p<0.05$ vs. control and $\mathrm{pc}-\mathrm{NC}$

pression in NSCLC tissues and cells, and its low expression was associated with high TNM stage and poor differentiation of NSCLC, but not with age, sex or lymphatic metastasis. Those results indicated that FENDRR might also be involved in tumorigenesis and progress of NSCLC, but not play a part in metastasis of NSCLC.

The Kaplan-Meier analysis showed FENDRR was a prognostic biomarker of overall survival for NSCLC patients, which corresponded to Xu's et al. report in gastric cancer [8]. Recent studies showed that several IncRNAs, including HMlincRNA717 and RGMB-AS1 [20, 21], were associated with overall survival of NSCLC patients, and could be prognostic factors for patients with NSCLC. Further penetrating research might provide a diagnosis target and prognostic biomarker for NSCLC.

In order to discuss the functions of FENDRR in NSCLC cells, the expression vector for FENDRR was transfected into $A 549$ cells to up-regulate the expression of FENDRR. Then, the results showed the up-regulation of FENDRR could inhibit the proliferation ability and advance cell apoptosis of A549 cells, which proved that FENDRR acted as a tumor suppressor gene in NSCLC cells. It was demonstrated that FENDRR could combine with polycomb repressive complex 2 (PRC2) and/ or TrxG/MLL complexes to promote the promoter methylation of target genes, and then silenced their expression [22, 23]. FENDER over-expression suppressed migration and invasion through downregulating the expression of FN1 and MMP2/MMP9 in gastric cancer cells [8].

In this study, the expression of FENDRR in cisplatin-resistant NSCLC patients' tissues and cell line was much lower than that in control tissues and cell line, which suggested that FENDRR depletion might be involved in chemotherapy resistance of NSCLC to cisplatin. Chemotherapy is an effective approach to prevent metastasis and recrudescence of NSCLC. But, the prognosis of NSCLC patients is still poor, because of chemotherapy resis- 
A

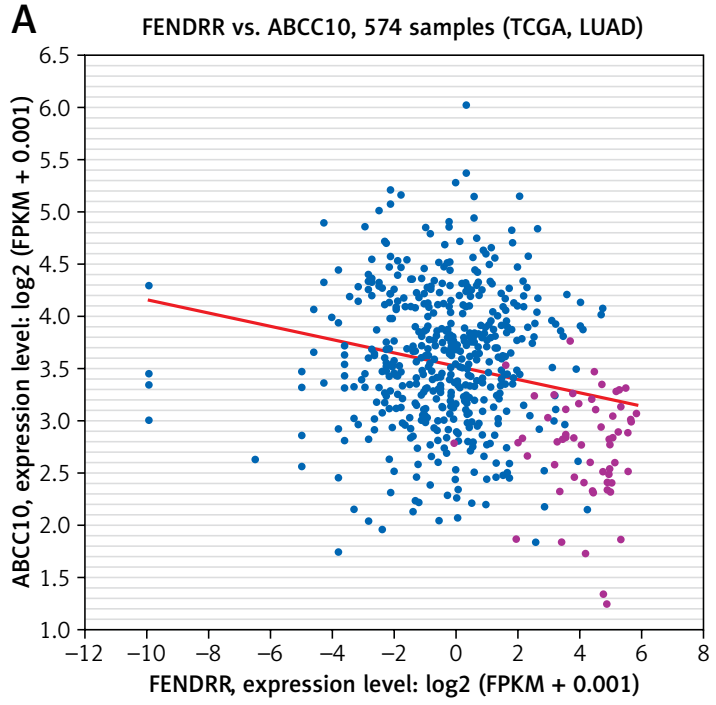

- Regression line $(y=-0.0635 x+3.5194)$

- FENDRR vs. ABCC10 $\left(r=-0.2167, p\right.$-value $\left.=1.59 \times 10^{-7}\right)$

Data Source: CHIPBase v2.0 project

\section{c}

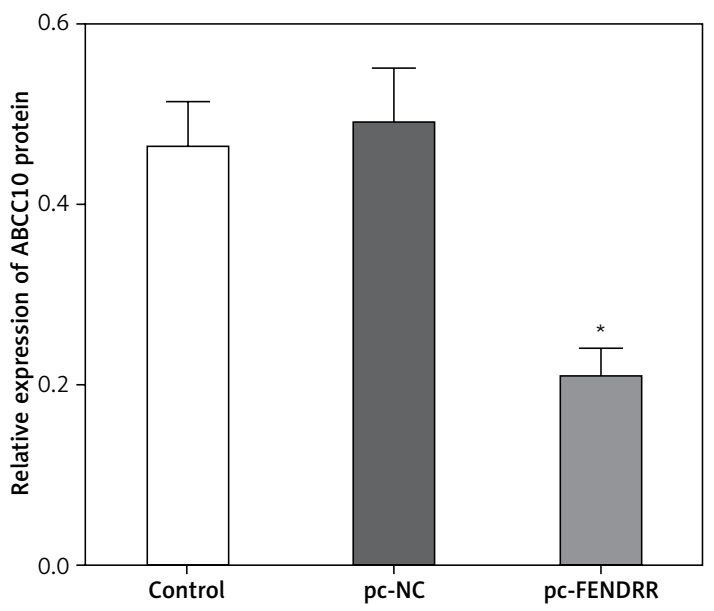

$\mathrm{ABCC} 10$

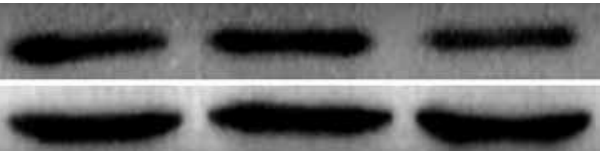

tance. Heretofore, nothing was known about the impact of FENDRR on chemotherapy resistance of NSCLC, especially to cisplatin. In this study, FENDRR over-expression decreased the IC50 for cisplatin in A549/DDP cells, promoted the cytotoxicity induced by DDP, and depressed chemotherapy resistance of A549/DDP cells to cisplatin. Recent papers reported that several IncRNAs participated in modulation of chemotherapy resistance in almost all malignant tumors [12, 24]. Shang et al. reported that silencing of long noncoding RNA UCA1 inhibited malignant prolifera-

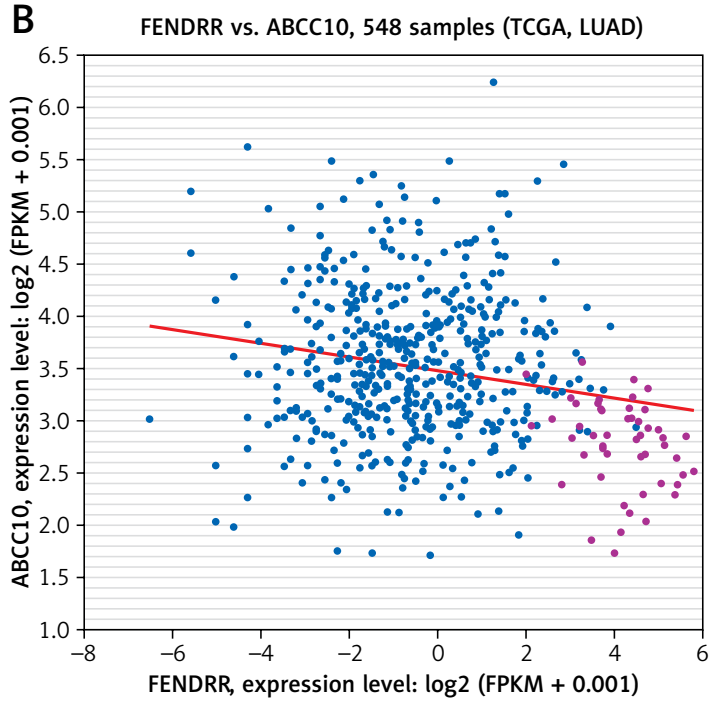

- Regression line $(y=-0.0665 x+3.4696)$

- FENDRR vs. ABCC10 $\left(r=-0.2014, p\right.$-value $\left.=2 \times 10^{-6}\right)$

Data Source: CHIPBase v2.0 project

Figure 4. Up-regulation of FENDRR inhibited the expression of ABCC10 in A549/DDP cells. The coexpression patterns between FENDRR and $A B C C 10$ in lung adenocarcinoma (A) and lung squamous cell carcinoma (B) was searched using the online server ChIPBase. C - FENDRR up-regulation inhibited the expression of ABCC10 in A549/DDP cells. ${ }^{*} p<0.05$ vs. control and $p c-N C$

tion and chemotherapy resistance to doxorubicin in gastric cancer [25]. HOTAIR, a well-known IncRNA, advanced cisplatin resistance through targeted silencing p21 expression in lung adenocarcinoma cells [11].

TCGA Pan-Cancer (PANCAN) analysis showed that the expression of FENDRR was negatively correlated with the expression of $A B C C 10$, and our western blot found FENDRR up-regulation inhibited the expression of ABCC10 in A549/DDP cells. ABCC10 is a member of the ATP-binding cassette $(A B C)$ transporter superfamily, which is also 
named multidrug resistance associated protein 7 (MRP7). ABCC10 is well characterized with drug resistance; it acts as an efflux pump to transport anticancer drugs out of the cells before they reach the cytosol $[26,27]$. Accordingly, we speculated that FENDRR depressed DDP resistance by inhibiting ABCC10 expression in A549/DDP.

In conclusion, IncRNA FENDRR shows low expression in NSCLC and is a prognostic biomarker of overall survival in NSCLC patients. FENDRR acts as a tumor-suppressing gene to inhibit the proliferation ability and chemotherapy resistance, and advance cell apoptosis of NSCLC cells, and this might offer a new therapy target for NSCLC.

\section{Acknowledgments}

This work was supported by the Doctoral Research Initiation Foundation of Liaoning Province (201601121).

\section{Conflict of interest}

The authors declare no conflict of interest.

\section{References}

1. Minguet J, Smith KH, Bramlage P. Targeted therapies for treatment of non-small cell lung cancer - recent advances and future perspectives. Int I Cancer 2016; 138: 2549-61.

2. Song Z, Zhang Y. Treatment and prognosis after progression in long-term responders to EGFR-tyrosine kinase inhibitor in advanced non-small cell lung cancer. Arch Med Sci 2016; 12: 107-11.

3. Carrizosa DR, Gold KA. New strategies in immunotherapy for non-small cell lung cancer. Transl Lung Cancer Res 2015; 4: 553-9.

4. Wu L, Jin L, Zhang W, Zhang L. Roles of long non-coding RNA CCAT2 in cervical cancer cell growth and apoptosis. Med Sci Monit 2016; 22: 875-9.

5. Pawar K, Hanisch C, Palma Vera SE, Einspanier R, Sharbati S. Down regulated IncRNA MEG3 eliminates mycobacteria in macrophages via autophagy. Sci Rep 2016; 6: 19416.

6. Wang Y, Wang H, Song T, et al. HOTAIR is a potential target for the treatment of cisplatin-resistant ovarian cancer. Mol Med Rep 2015; 12: 2211-6.

7. Li P, Zhang G, Li J, et al. Long noncoding RNA RGMB-AS1 indicates a poor prognosis and modulates cell proliferation, migration and invasion in lung adenocarcinoma. PLoS One 2016; 11: e0150790.

8. Xu TP, Huang MD, Xia R, et al. Decreased expression of the long non-coding RNA FENDRR is associated with poor prognosis in gastric cancer and FENDRR regulates gastric cancer cell metastasis by affecting fibronectin 1 expression. J Hematol Oncol 2014 2014; 7: 63.

9. Li Q, Wu C, Song G, et al. Genome-wide analysis of long noncoding RNA epression profiles in human Xuanwei lung cancer. Clin Lab 2015; 61: 1515-23.

10. Hong Y, Shang C, Xue YX, YH. Silencing of Bmi-1 gene enhances chemotherapy sensitivity in human glioblastoma cells. Med Sci Monit 2015; 21: 1002-7.

11. Liu Z, Sun M, Lu K, et al. The long noncoding RNA HOTAIR contributes to cisplatin resistance of human lung adeno- carcinoma cells via downregualtion of p21(WAF1/CIP1) expression. PLoS One 2013; 8: e77293.

12. Shang C, Guo Y, Zhang H, Xue YX. Long noncoding RNA HOTAIR is a prognostic biomarker andinhibits chemosensitivity to cisplatin in bladder transitional cell carcinoma. Cancer Chemother Pharmacol 2016; 77: 507-13.

13. Ge $Y$, Yan X, Jin Y, et al. fMiRNA-192 and miRNA-204 directly suppress IncRNA HOTTIP and interrupt GLS1-mediated glutaminolysis in hepatocellular carcinoma. PLoS Genet 2015; 11: e1005726.

14. Chen Y, Ni H, Zhao Y, et al. Potential role of IncRNAs in contributing to pathogenesis of intervertebral disc degeneration based on microarray data. Med Sci Monit 2015; 21: 3449-58.

15. Yu X, Li Z. Long non-coding RNA growth arrest-specific transcript 5 in tumor biology. Oncol Lett 2015; 10: 1953-8.

16. Ye N, Wang B, Quan ZF, et al. Functional roles of long non-coding RNA in human breast cancer. Asian Pac J Cancer Prev 2014; 15: 5993-7.

17. Huang $\mathrm{Y}, \mathrm{Du} J, \mathrm{Mi} \mathrm{Y}$, et al. Long non-coding RNAs contribute to the inhibition of proliferation and EMT by pterostilbene in human breast cancer. Front Oncol 2018; 8: 629.

18. Liu Z, Xie D, Zhang H. Long noncoding RNA neuroblastoma-associated transcript 1 gene inhibits malignant cellular phenotypes of bladder cancer through miR-21/ SOCS6 axis. Cell Death Dis 2018; 9: 1042.

19. Kraus TF, Greiner A, Guibourt V, Lisec K, Kretzschmar HA. Identification of stably expressed IncRNAs as valid endogenous controls for profiling of human non-small cell lung cancer. J Cancer 2015; 6: 111-9.

20. Li P, Li J, Yang R, et al. Study on expression of IncRNA RGMB-AS1 and repulsive guidance molecule $b$ in nonsmall cell lung cancer. Diagn Pathol 2015; 10: 63.

21. Xie X, Liu HT, Mei J, et al. LncRNA HMlincRNA717 is down-regulated in non-small cell lung cancer and associated with poor prognosis. Int J Clin Exp Pathol 2014; 7: 8881-6.

22. Grote P, Herrmann BG. The long non-coding RNA Fendrr links epigenetic control mechanisms to gene regulatory networks in mammalian embryogenesis. RNA Biol 2013; 10: 1579-85.

23. Grote P, Wittler L, Hendrix D, et al. The tissue-specific IncRNA Fendrr is an essential regulator of heart and body wall development in the mouse. Dev Cell 2013; 24: 206-14.

24. Xie D, Zhang H, Shang C. Long non-coding RNA CDKN2B antisense RNA 1 gene inhibits gemcitabine sensitivity in bladder urothelial carcinoma. J Cancer 2018; 9: 2160-6.

25. Shang C, Guo Y, Zhang J, Huang B. Silence of long noncoding RNA UCA1 inhibits malignant proliferation and chemotherapy resistance to adriamycin in gastric cancer. Cancer Chemother Pharmacol 2016; 77: 1061-7.

26. Xie T, Geng J, Wang Y, et al. FOXM1 evokes 5-fluorouracil resistance in colorectal cancer depending on $\mathrm{ABCC} 10$. Oncotarget 2017; 8: 8574-89.

27. Krizkova V, Dubova M, Susova S, et al. Protein expression of ATP-binding cassette transporters $\mathrm{ABCC} 10$ and ABCC11 associates with survival of colorectal cancer patients. Cancer Chemother Pharmacol 2016; 78: 595-603. 